\section{Zusammenfassung}

Die Forderung nach mehr Interdisziplinarität gehört seit Jahrzehnten zu den Standardfloskeln der Wissenschaftspolitik. Bemerkenswerterweise hat sie nicht dazu geführt, der fortschreitenden Spezialisierung der Wissenschaften Einhalt zu gebieten. Ganz im Gegenteil verhält es sich so, dass Interdisziplinarität, gerade weil sie wissenschaftliche Innovation befördert, auch die weitere wissenschaftliche Spezialisierung unterstützt. Interdisziplinarität kann Missverständnissen Vorschub leisten, eine Tendenz zu unverbindlichem Gerede hervorrufen und u. U. selbst fachliche Standards in Gefahr bringen. Gelingende Interdisziplinarität setzt deshalb mindestens dreierlei voraus: Reflexion auf die fachlichen Standards der eigenen Disziplin, frühzeitige Einigung über die eigentliche Problemstellung und schließlich den unbedingten Willen zu sprachlicher Klarheit.

Professor Dr. Friedrich Pukelsheim und Professor Dr. Matthias Rossi, Augsburg*

\title{
Wahlsystemnahe Optionen zur Vermeidung negativer Stimmgewichte
}

\begin{abstract}
Das BVerfG hat 2008 zwei Bestimmungen des Bundeswahlgesetzes insoweit für verfassungswidrig erklärt, als sie negative Stimmgewichte bewirken können. Dem Bundesgesetzgeber ist aufgegeben, bis zum 30. Juni 2011 eine verfassungsgemäße Regelung zu treffen. Vor diesem Hintergrund illustriert der Beitrag den monierten Defekt anhand der Bundestagswahl 2009 und diskutiert Optionen, die negative Stimmgewichte ausschließen und doch eine mit der Personenwahl verbundene Verhältniswahl fortschreiben, die sich seit mehr als einem halben Jahrhundert bewährt hat.

\section{Verfassungsgerichtlicher Gesetz- gebungsauftrag und verfassungsrechtlicher Gesetzgebungsrahmen}

Das BVerfG hat mit Urteil vom 3.7. 2008 das Auftreten von negativen Stimmgewichten im Bundeswahlgesetz für verfassungswidrig erklärt und dem Gesetzgeber aufgegeben, das Gesetz bis zum 30.6. 2011 entsprechend zu korrigieren. ${ }^{1}$ Die gesetzgeberische Gestaltungsfreiheit ${ }^{2}$ reicht von Feinkorrekturen des bestehenden Wahlsystems, die das überkommene und grundsätzlich auch bewährte bundesdeutsche Wahlrecht weitgehend bestehen ließen, bis hin zu einer grundsätzlichen Neugestaltung des gesamten Wahlsystems. Das $B \operatorname{VerfG}$ hat diesen umfassenden Gestaltungsspielraum des Gesetzgebers explizit hervorgehoben und selbst verschiedene Möglichkeiten der Neuregelung angedeutet. ${ }^{3}$ Allerdings formt die verfassungsrechtliche Bindung des Gesetzgebers quasi ein begrenzendes Dreieck um sein politisches Ermessen: Als erster Eckpunkt sind die grundrechtsgleich ausgestalteten und subjektive Rechte vermittelnden Wahlrechtsgrundsätze des Art. 38 Abs. 1 Satz 1 GG zu beachten. Einen zweiten Eckpunkt markiert die aus Art. 21 Abs. 1 i.V. mit Art. 3 Abs. 1 GG abgeleitete (Chancen-)

\footnotetext{
* Die Autoren sind Ordinarien an der Universität Augsburg, Friedrich Pukelsheim für Stochastik und ihre Anwendungen an der MathematischNaturwissenschaftlichen Fakultät, Matthias Rossi für Staats- und Verwaltungsrecht, Europarecht sowie Gesetzgebungslehre an der Juristischen Fakultät.

$1 B \operatorname{VerfGE} 121,266$, Ls. 1 u. 2. Zu Gesetzgebungsaufträgen des BVerfG vgl. allgemein Brandner, Gesetzesänderung, 2004, S. $189 \mathrm{ff}$.

2 Hierzu grundlegend Meßerschmidt, Gesetzgebungsermessen, 2000, passim, in Bezug auf verfassungsgerichtliche Eingrenzung insbesondere S. $713 \mathrm{ff}$.

3 BVerfGE 121, 266 (307).
}

Gleichheit der politischen Parteien. Als dritter Eckpunkt kommt die aus Art. 38 Abs. 1 Satz 2 GG folgende (Status-) Gleichheit der gewählten Abgeordneten hinzu. Über diese Verfassungsvorgaben wölben sich zudem die allgemeinen rechtsstaatlichen und demokratischen Anforderungen: Das Wahlrecht muss in sich stimmig, folgerichtig ${ }^{4}$ und dem Wähler klar und verständlich, ${ }^{5}$ kurz, es muss transparent sein. ${ }^{6}$

Vor dem Hintergrund dieser komplexen Rahmenbedingungen - aber auch vor dem Hintergrund der nicht nur legitimierenden, ${ }^{7}$ sondern zugleich integrierenden Bedeutung ${ }^{8}$ von Wahlen und damit des Wahlsystems - erscheint es sinnvoll, das Wahlsystem im Grundsatz zu bewahren und nur die verfassungswidrigen Einzelelemente systemnah zu korrigieren. Zwar wäre es theoretisch und auch verfassungsrechtlich möglich, das Wahlrecht zum Bundestag vollständig neu zu regeln. Der Gesetzgeber ist insoweit frei, grundsätzlich andere Wege zu gehen als im geltenden BWahlG. Nicht zuletzt aber vor dem Hintergrund, dass jegliche Novellierung des als materielles Verfassungsrecht $\mathrm{zu}$ bewertenden Wahlrechts politisch auf eine breitere Grundlage als bloß auf die Kanzlermehrheit im Bundestag gestellt sein sollte und für grundlegende Änderungen im 17. Bundestag die politischen, jedenfalls aber zunehmend die zeitlichen Voraussetzungen fehlen, soll im Folgenden ausgelotet werden, mit welchen minimalen Korrekturen des geltenden Wahlrechts negative Stimmgewichte vermieden werden können. ${ }^{9}$

Nah am bisherigen System bleiben solche Alternativen, die den Wählern zwei Stimmen belassen $(\mathbb{S} 4$ BWahlG) und unproblematische Fälle so behandeln wie bisher. Die Umrechnung der Stimmen in Sitze bedarf nur dann einer Anpassung, wenn problematische Fälle wie ein Auftreten negativer Stimmgewichte ausgeschlossen werden sollen. Die Bundestagsabgeordneten werden nach den Grundsätzen einer mit der Personenwahl verbundenen Verhältniswahl gewählt.

\footnotetext{
4 Zur Folgerichtigkeit als allgemeines Postulat kritisch Rossi, in: Koch/ Rossi (Hrsg.), Kodifikation in Europa, 2010, im Erscheinen.

5 So mittelbar $B \operatorname{Verf} G E$ 121, 266 (316): „normenklare und verständliche Grundlage“.

6 BVerfGE 123, 39 (69) = JZ 2009, 566 mit Anm. S. Schiedermair

7 Vgl. Stern, Staatsrecht, Bd. I, 2. Aufl. 1984, S. 293 f.

8 Vgl. BVerfGE 6, 84 (92f.); 24, 300 (341) = JZ 1969, 557 (dazu Randelshofer JZ 1969, 533); 51, 222 (236); 71, 81 (97); 95, 408 (418ff.); sowie vor allem Smend, in: ders., Staatsrechtliche Abhandlungen, 2. Aufl. 1968, S. 119 (154).

9 Verwandte Lösungsmöglichkeiten, wenn auch anders akzentuiert: $H$. Meyer DVBl 2009, $137 \mathrm{ff}$.
} 
Die Zuteilung der Sitze an die Wahlkreis- und Listenbewerber erfolgt in einem dreistufigen Verfahren. Erstens werden im Wege der sogenannten Oberzuteilung 598 Sitze den Parteien im Verhältnis ihrer bundesweiten Zweitstimmenerfolge zugeteilt. Zweitens werden diese Parteisitze in sogenannten Unterzuteilungen pro Partei an ihre Landeslisten im Verhältnis der Zweitstimmen weiter gereicht. Drittens werden von den so ermittelten Verhältnissitzen die Direktmandate abgerechnet. Nur die restlichen Mandate werden mit Listenbewerbern besetzt. Wenn die mittels Verhältnisrechnung einer Parteilandesliste zugeteilten Sitze nicht für alle ihre Wahlkreissieger reichen, kommen Zusatzsitze zu Stande und der Bundestag wird über die Ausgangszahl 598 hinaus um sogenannte Überhangmandate größer.

Negative Stimmgewichte können entstehen, weil das System eine Personenwahl- und eine Verhältniswahlkomponente besitzt und die föderale Untergliederung in sechzehn Bundesländer berücksichtigt. Die „Reparaturoptionen“ lassen sich danach ordnen, welcher dieser drei Aspekte dominieren soll. Option P behandelt die Personenwahlkomponente als sakrosankt (III.), Option V die Verhältniswahlkomponente (IV.) und Option F die Einbeziehung der föderalen Staatsgliederung (V.). Zuvor sei am Beispiel der Wahl zum 17. Bundestag im September 2009 illustriert, wie das geltende Bundeswahlrecht die von den Wählern abgegebenen Stimmen in Mandate umrechnet und wie dabei negative Stimmgewichte auftreten können (II.).

\section{Zahlen und Besonderheiten der Wahl zum 17. Bundestag im September 2009}

Bei der Wahl traten zwei Besonderheiten auf, die vom $B$ VerfG bei seiner Entscheidung vom 3.7. 2008 nicht in den Blick genommen wurden. Zum einen kam es erstmals schon auf Bundesebene zu Überhangmandaten, sie entfielen auf die unverbundene Landesliste der CSU. Zum anderen erreichte die CDU mit 173 Wahlkreissiegen dieselbe Mandatszahl wie in der Oberzuteilung. Ein einziges zusätzliches CDU-Direktmandat hätte erstmals einer Partei, die mit verbundenen Landeslisten antritt, auf Bundesebene ein Überhangmandat beschert. ${ }^{10}$ Die Analyse des Wahlsystems sollte deshalb nicht nur praktisch-empirische, sondern auch theoretisch-denkbare Fallgestaltungen im Auge behalten.

Für die Wahl 2009 waren 62168489 Deutsche wahlberechtigt. Davon blieben 18162914 der Wahl fern, 44005575 Wähler nahmen teil..1 Von diesen gaben 634385 ihre Zweitstimme ungültig ab, 43371190 Zweitstimmen waren gültig. Darunter gab es 2606902 Wähler, deren Zweitstimmen das Gesetz die Zuteilungsberechtigung vorenthielt. ${ }^{12}$ Die verbleibenden 40764288 zuteilungsberechtigten Zweitstimmen bilden die Grundlage für die Verhältnisrechnung.

10 Noch bei der Bundestagsinnenausschussanhörung im Mai 2009 wurden beide Besonderheiten allseits für unwahrscheinlich gehalten; siehe http://www.uni-augsburg.de/pukelsheim/2009Berlin.

11 Gisart Statistisches Bundesamt, Wirtschaft und Statistik 11/2009, 1063 (1076).

12 Eine Zweitstimme ist zuteilungsberechtigt, falls sie gültig ist und für eine Partei abgegeben wird, die mindestens fünf Prozent der gültigen Zweitstimmen erhält (Fünfprozenthürde) oder mindestens drei Direktmandate erringt (Grundmandatsklausel) oder eine nationale Minderheit vertritt (Minderheitenprivileg). Zudem bleibt einer Zweitstimme die Zuteilungsberechtigung versagt, falls ihre zugehörige Erststimme zum Mandatsgewinn eines Wahlkreiskandidaten beiträgt, der parteilos ist oder für dessen Partei im betreffenden Bundesland keine Landesliste zugelassen ist.

\section{Das Divisorverfahren mit Standardrundung (Sainte-Laguë/Schepers)}

Seit der Novellierung des Bundeswahlgesetzes im März 2008 wird für die Umrechnung der Zweitstimmen in Mandate das sogenannte Divisorverfahren mit Standardrundung verwendet. Der das Verfahren bestimmende Divisor dient als Wahlschlüssel, der den Zugang zum Ergebnis eröffnet. Als Wahlschlüssel kommt jede Zahl aus einem Bereich in Frage, der durch den Übergang zur nächstgrößeren oder nächstkleineren Gesamtsitzzahl abgegrenzt ist. ${ }^{13}$ Die auf den ersten Blick innerhalb gewisser Grenzen gegebene Beweglichkeit des Wahlschlüssels mag als Willkür erscheinen, wird aber durch anschließende Rundung der errechneten Quotienten neutralisiert. So ist sichergestellt, dass die sich ergebende Sitzzuteilung eindeutig ist. Rechtliche Relevanz hat allein die Eindeutigkeit des Ergebnisses. Dagegen ist irrelevant, dass das Ergebnis angesichts der Beweglichkeit des Wahlschlüssels auf unterschiedliche Weisen berechnet werden kann. Bei der Wahl 2009 liegt der Wahlschlüssel im Bereich zwischen 68194.46 und $68196.51 .{ }^{14}$ Schaubild 1 rechnet mit dem mittigen Wert 68196.

In Schaubild 1 werden die Stimmenzahlen durch den Wahlschlüssel geteilt und die resultierenden Quotienten standardmäßig zur Sitzzahl gerundet. Die Standardrundung geht so vor, wie es im kaufmännischen Bereich die Regel ist: Ein Bruchteilsrest kleiner als ein Halbes wird abgerundet. Ist er größer als ein Halbes, wird aufgerundet. Im Schaubild sind so viele Nachkommastellen ausgewiesen, dass die Rundungsrichtung eindeutig erkennbar wird. Ein prägnanter Satz reicht aus, um die Sitzzuteilung zu beschreiben: Auf je 68196 Zweitstimmen entfällt rund ein Verhältnissitz.

Die SPD-Stimmen sind mit den ihnen zugeteilten 146 Sitzen von Proporzpech betroffen. Ihr Quotient 9990488/ $68196=146497$ verpasst knapp die nächste Rundungsschwelle (146.5). Ein Wahlschlüssel kleiner als 9990488 / $146.5=68194.46$ hätte $\mathrm{zu}$ einem Bruchteilsrest größer als ein Halbes und zur Aufrundung geführt. Dies ist aber ausgeschlossen, weil dann 599 Sitze zugeteilt werden, obwohl nur 598 anstehen. Oder anders ausgedrückt: Hätte über die 598 Ausgangssitze hinaus ein weiterer Sitz zur Verfügung gestanden, wäre er den SPD-Wählern zu Gute gekommen. Die CSU-Stimmen sind bei 42 Sitzen von Proporzglück bedacht, weil sie mit Quotient 2830238/68196 = 41502 um ein Weniges die letzte Rundungsschwelle übertreffen (41.5). Bei einem Wahlschlüssel größer als 2830 238/41.5 = 68198.51 wäre der Bruchteilsrest unter ein Halbes gerutscht und abgerundet worden. Dies kann aber keinen Bestand haben, weil dann nur 597 Sitze verteilt werden. Oder anders herum: Der letzte der 598 Verhältnissitze entfällt auf die CSU-Stimmen. Allerdings entstehen drei Überhangmandate, da die CSU mit 45 Wahlkreisen drei Mandate mehr erworben hat, als ihr nach der Oberzuteilung zustehen.

Die Transparenz des Lösungssatzes „Auf je 68196 Zweitstimmen entfällt rund ein Sitz“ ist eine bemerkens-

$13 \sqrt{ } 6$ Abs. 2 Sätze 5-7 BWahlG enthalten nähere Vorgaben zur Bestimmung des Wahlschlüssels. Nähere Erläuterungen dazu in: Bericht der Wahlkreiskommission für die 16. Wahlperiode des Deutschen Bundestags, BT-Drs. 16/4300 vom 24. 1. 2007, Anlage 1d.

14 Die Schwellenwerte entstehen dadurch, dass die Stimmenzahlen durch die Teiler $0.5,1.5,2.5, \ldots, 41.5, \ldots, 146.5$, etc. dividiert werden. Dieses Vorgehen ist dasselbe wie im früheren Divisorverfahren mit Abrundung (D’Hondt/Hagenbach-Bischoff), außer dass dort die Teiler 1, 2, 3, .., 42, ..., 147 etc. benutzt wurden. Die Schwellenwerte werden traditionell auch Höchstzahlen genannt. In dieser Diktion ist 68198.51 die letzte Höchstzahl, auf die ein Sitz entfällt, und 68194.46 die erste, die leer ausgeht. 


\begin{tabular}{|lrcr|}
\hline \multicolumn{4}{|l|}{ Schaubild 1: Oberzuteilung im Bundesgebiet } \\
\hline 27. September 2009 & $\begin{array}{r}\text { Zweit- } \\
\text { stimmen }\end{array}$ & $\begin{array}{c}\text { Quotient } \\
\frac{\text { Zweistinmen }}{68196}\end{array}$ & $\begin{array}{r}\text { Verhältnis- } \\
\text { sitze }\end{array}$ \\
\hline CDU & 11828277 & 173.4 & 173 \\
\hline SPD & 9990488 & 146.497 & 146 \\
\hline FDP & 6316080 & 92.6 & 93 \\
\hline LINKE & 5155933 & 75.6 & 76 \\
\hline GRÜNE & 4643272 & 68.1 & 68 \\
\hline CSU & 2830238 & 41.502 & 42 \\
\hline Summe & 40764288 & & 598
\end{tabular}

Auf je 68196 Zweitstimmen entfällt rund ein Verhältnissitz.

werte Stärke des Divisorverfahrens mit Standardrundung. ${ }^{15}$ Aber verfassungsrechtlich ausschlaggebend ist die besondere Vereinbarkeit des Verfahrens mit dem Grundsatz der Wahlgleichheit. Das $B \operatorname{VerfG}$ präzisiert und stilisiert diesen Grundsatz zur Erfolgswertgleichheit der Wählerstimmen. ${ }^{16}$ Mit dieser wählerbezogenen Gleichheitsnorm harmoniert das Divisorverfahren mit Standardrundung in ganz hervorragendem Maße. ${ }^{17}$

Die hohe Übereinstimmung von Gleichheitsanspruch und Verfahrenswirklichkeit ist zunächst auf die Verhältnisrechnung beschränkt. Nach dem geltenden Wahlrecht treten neben die Verhältniskomponente aber noch Elemente der Personenwahl. Das Zusammenspiel dieser beiden Komponenten kann zu Folgen führen, die das BVerfG für verfassungswidrig hält, zu negativen Stimmgewichten. Sie können im Rahmen einer Unterzuteilung auftreten, aber unter bestimmten Umständen auch im Rahmen der Oberzuteilung.

\section{Negative Stimmgewichte in einer Unterzuteilung}

Bei der Unterzuteilung der bundesweiten Sitze einer Partei an ihre Landeslisten können negative Stimmgewichte dort auftreten, wo die Erststimmenerfolge berücksichtigt werden. Fallen die Direktmandatsgewinne einer Partei geringer aus als die der Landesliste zugeteilten Verhältnissitze, kommen alle Wahlkreissieger im Lande zum Zuge und die restlichen Verhältnissitze werden aus der Landesliste besetzt. Dieser Fall ist unproblematisch.

Gewinnt aber eine Partei mehr Direktmandate als Verhältnissitze, wird eine Unterschiedszahl ins Leben gerufen, die den Kern des Problems darstellt. ${ }^{18}$ Denn da das Wahlgesetz allen Wahlkreissiegern ein Parlamentsmandat zuweist und die Verhältnisrechnung nicht genügend Sitze bereit stellt, werden Zusatzsitze nötig. In Höhe der Unterschiedszahl entstehen Überhangmandate und der Bundestag wird über die Ausgangszahl von 598 Sitzen hinaus größer. Schon Geyerhabn ${ }^{19}$ erwog, dass die Wahlkreissiege die Verhältnissitze übertreffen können, sah darin aber nur eine seltene

15 Das Verfahren benutzt einen beweglichen Wahlschlüssel und ein festes Rundungsverfahren. Dagegen beruht das frühere Quotenverfahren mit Ausgleich nach größten Resten (Hare/Niemeyer) auf einem festen Wahlschlüssel und einem beweglichen Rundungsverfahren. Ein Verfahren, das einen festen Wahlschlüssel mit einem festen Rundungsverfahren kombiniert, um immer die vorgegebene $Z$ ahl von Sitzen zuzuteilen, gibt es nicht. 16 Vgl. ausführlich BVerfGE 95, 408 (418), wo das BVerfG seine Rechtsprechung zu Differenzierungen des Erfolgswerts der Wählerstimmen zusammenfasst.

17 Pukelsheim DVBl 2008, 889 (895); und W. Schreiber DVBl 2006, 529 (534).

$18 \rrbracket 6$ Abs. 5 BWahlG.

19 Geyerhahn, Das Problem der verhältnismäßigen Vertretung, Wiener Staatswissenschaftliche Studien, Bd. 3 Heft 4, 1902, S. 29.
Eventualität. Vor 1980 traten in Bundestagswahlen Überhangmandate in der Tat nur unregelmäßig auf. Seitdem ist die seltene Eventualität zum Regelfall geworden.

Soweit es ein bestimmtes Bundesland und eine bestimmte Partei betrifft, bezeichne $d$ die Zahl der Direktmandate und $v$ die Zahl der Verhältnissitze. Die Unterschiedszahl $d-v$ wurde lange Zeit einseitig mit Sicht auf die $d$ Direktmandate beurteilt. Wenn mehr Wahlkreise gewonnen werden, wächst $d$ und damit auch die Unterschiedszahl. Mehr Wahlkreisgewinne bedeuten mehr Erfolg bei den Wählern, mehr Wahlerfolg rechtfertigt mehr Parlamentsmandate. Dass diese Mandate zur Ausgangssitzzahl hinzutreten und nicht in die Verhältnisrechnung eingebettet sind, erscheint dann als Eigenheit eines Systems, das eine mit der Personenwahl verbundene Verhältniswahl verwirklicht.

Meyer war der erste, der auch die Verhältnissitze $v$ in den Blick nahm. ${ }^{20}$ Die Unterschiedszahl $d-v$ wächst auch dann, wenn die Zahl der Verhältnissitze $v$ kleiner wird. Weniger Verhältnissitze kommen durch weniger Erfolg bei den Zweitstimmen zu Stande. Ein Verhältnissitz, der in einem Land frei wird, kann aber das Sitzkontingent in einem anderen Land vergrößern. Hat die Partei in einem Land schon Überhangmandate sicher, dann bleibt der Verlust von Zweitstimmen dort ohne Wirkung, aber in einem anderen Land kann ein Verhältnissitz hinzukommen. Weniger Zweitstimmen können so zu einem Sitzgewinn für die Partei insgesamt führen.

Aus Sicht der Gesamtpartei erscheint dies profitabel, wenn auch nur schwer steuerbar. Aus Sicht derjenigen Wähler, die das Wahlrecht nicht nur als „black box“ betrachten, sondern die Wirkung ihrer Stimmen verstehen wollen, stellt sich diese Gegenläufigkeit dagegen als Desorientierung dar. Sie verunsichert und stellt die Legitimations- und Integrationskraft des Wahlsystems in Frage.

Schaubild 2 zeigt die Unterzuteilung von 173 Sitzen an die CDU-Landeslisten. Auf je 69000 Zweitstimmen entfällt rund ein Verhältnissitz. Nach Abrechnung der Direktmandate entstehen 21 Überhangmandate, davon vier in Sachsen. Hier lassen sich die Auswirkungen negativer Stimmgewichte sichtbar machen. Der Verlust von 15000 CDU-Zweitstimmen in Sachsen hätte das CDU-Bundeskontingent gleich gelassen, aber Niedersachsen einen Sitz mehr zugeteilt und Sachsen einen weniger. ${ }^{21}$ Bei sechzehn Direktmandaten wäre bei der sächsischen CDU ein weiteres Überhangmandat hinzugekommen, bundesweit wären also 22 statt 21 entstanden. Der Verlust an Zweitstimmen hätte also den Gewinn eines Sitzes für die CDU-Fraktion nach sich gezogen, von $173+$ $21=194$ auf $173+22=195$ Sitze.

Diese Eigenheit hat nichts mit einer parteipolitischen Ausrichtung $\mathrm{zu}$ tun. Genauso hätte der Verlust von 600 SPD-Zweitstimmen in Bremen zu einem Sitzgewinn für die SPD-Fraktion geführt. ${ }^{22}$ Die Gegenläufigkeit von Stimmenzahl und Sitzzuteilung, die mit dem Begriff negative Stimmgewichte umschrieben wird, hat das $B \operatorname{Verf} G$ als verfassungswidrig verworfen. ${ }^{23}$

20 H. Meyer KritV 1994, 312 (321)

21 Bei dann 785898 Zweitstimmen für die CDU in Sachsen, mit Divisor 68400 .

22 Eine detailreiche Analyse der Dresdner Nachwahl 2005 bietet Behnke Politische Vierteljahresschrift 49 (2008), 695 (697). Weitere Beispiele mit den Zahlen der Wahl 2009 bei Lübbert Zeitschrift für Parlamentsfragen (Zparl) 2/2010, 278 (283). Zur Verursachung negativer Stimmgewichte durch Beteiligungsquoren bei Volksabstimmung Jung, in: Feld/Huber/Jung/Welzel/ Wittreck (Hrsg.), Jahrbuch für direkte Demokratie 2009, S. 40 (61).

$23 B \operatorname{VerfGE} 121,266$ (Ls.) 


\begin{tabular}{|c|c|c|c|c|c|}
\hline 27. September 2009 & $\begin{array}{l}\text { Direkt- } \\
\text { mandate }\end{array}$ & $\begin{array}{l}\text { Zweit- } \\
\text { stimmen }\end{array}$ & $\begin{array}{l}\text { Quotient } \\
\frac{\text { Zweitstimmen }}{69000}\end{array}$ & $\begin{array}{r}\text { Verhältnis- } \\
\text { sitze }\end{array}$ & $\begin{array}{l}\text { Überhang- } \\
\text { mandate }\end{array}$ \\
\hline Schleswig-Holstein & 9 & 518457 & 7.51 & 8 & 1 \\
\hline Mecklenburg-Vorpommern & 6 & 287481 & 4.2 & 4 & 2 \\
\hline Hamburg & 3 & 246667 & 3.6 & 4 & \\
\hline Niedersachsen & 16 & 1471530 & 21.3 & 21 & \\
\hline Bremen & 0 & 80964 & 1.2 & 1 & \\
\hline Brandenburg & 1 & 327454 & 4.7 & 5 & \\
\hline Sachsen-Anhalt & 4 & 362311 & 5.3 & 5 & \\
\hline Berlin & 5 & 393180 & 5.7 & 6 & \\
\hline Nordrhein-Westfalen & 37 & 3111478 & 45.1 & 45 & \\
\hline Sachsen & 16 & 800898 & 11.6 & 12 & 4 \\
\hline Hessen & 15 & 1022822 & 14.8 & 15 & \\
\hline Thüringen & 7 & 383778 & 5.6 & 6 & 1 \\
\hline Rheinland-Pfalz & 13 & 767487 & 11.1 & 11 & 2 \\
\hline Baden-Württemberg & 37 & 1874481 & 27.2 & 27 & 10 \\
\hline Saarland & 4 & 179289 & 2.6 & 3 & 1 \\
\hline Summe & 173 & 11828277 & & 173 & 21 \\
\hline
\end{tabular}

\begin{tabular}{|c|c|c|c|c|c|}
\hline \multicolumn{6}{|c|}{ Schaubild 3: CSU-Stimmenverlust und CDU-Sitzgewinn } \\
\hline Fiktives Szenario & $\begin{array}{c}\text { Direkt- } \\
\text { mandate }\end{array}$ & $\begin{array}{r}\text { Zweit- } \\
\text { stimmen }\end{array}$ & $\begin{array}{l}\text { Quotient } \\
\frac{\text { Zweitstimmen }}{68183}\end{array}$ & $\begin{array}{r}\text { Verbältnis- } \\
\text { sitze }\end{array}$ & $\begin{array}{l}\text { Überhang } \\
\text { mandate }\end{array}$ \\
\hline $\mathrm{CDU}(+2000)$ & 173 & 11830277 & 173.508 & 174 & 21 \\
\hline $\operatorname{SPD}(-2000)$ & 64 & 9988488 & 146.495 & 146 & \\
\hline FDP & 0 & 6316080 & 92.6 & 93 & \\
\hline LINKE & 16 & 5155933 & 75.6 & 76 & \\
\hline GRÜNE & 1 & 4643272 & 68.1 & 68 & \\
\hline $\operatorname{CSU}(-68000)$ & 45 & 2762238 & 40.51 & 41 & 4 \\
\hline Summe & 299 & 40696288 & & 598 & 25 \\
\hline
\end{tabular}

\section{Negative Stimmgewichte in der Oberzuteilung}

Auch die Oberzuteilung ist für negative Stimmgewichte anfällig. Bis zur Wahl 2009 erschien dies als vollkommen unrealistisch. Dementsprechend hat sich auch das BVerfG nicht zu diesem Problem geäußert. Der Gesetzgebungsauftrag bezieht sich deshalb bei enger Auslegung nur auf die Beseitigung negativer Stimmgewichte in den Unterzuteilungen. Freilich steht es dem Gesetzgeber offen, sie auch in der Oberzuteilung auszuschließen. Konfrontiert ist er mit der Problematik jedenfalls insofern, als erstmals Überhandmandate auf die CSU entfallen und damit die thematisierte Unterschiedszahl auch in der Oberzuteilung an Bedeutung gewinnt.

Das Wahlergebnis 2009 verursacht keine Probleme, wie aus Schaubild 1 ersichtlich wird. Hätten etwas weniger Wähler ihre Zweitstimme der CSU gegeben, so ginge der dadurch freiwerdende CSU-Verhältnissitz an die SPD, die mit Quotient 146497 sichtbar die Aspirantin auf den nächsten verfügbaren Sitz ist. Weniger Stimmen für eine Partei (CSU) führen zur Stärkung einer konkurrierenden Partei (SPD) - das erscheint als Logik politischer Repräsentation recht und billig. Anzumerken ist allerdings, dass die CSU nicht schwächer vertreten wäre, da ihre 45 Wahlkreissiege unangetastet bleiben.
Anders sähe die Lage aus, wenn der CSU-Sitz zum politischen Freund wanderte. Zur Konstruktion eines solchen fiktiven Szenarios seien in Niedersachsen 2000 Stimmen von der SPD zur CDU transferiert. Mit dieser Umgewichtung ginge die Aspirantur auf den nächsten Sitz zur CDU über. Wie Schaubild 3 verdeutlicht, würde der Verlust von 68000 CSU-Stimmen - was fast den Divisor 68183 erreichen und einen Sitzverlust bedeuten würde - zu einem Sitzgewinn der niedersächsischen CDU führen. Die CDU/CSU-Fraktion würde um ein Mitglied gestärkt, von vorher $173+21+45=$ 239 auf jetzt $174+21+45=240$ Sitze.

Das fiktive Szenario erzeugt dieselbe verkehrte Welt wie negative Stimmgewichte. CSU-Anhänger könnten ihrer Partei die Zweitstimme verweigern, um die Unionsfraktion insgesamt zu stärken. Anhänger gegnerischer Parteien mögen erwägen, mit ihren Zweitstimmen zur CSU überzulaufen, um den Sitzgewinn der Unionsfraktion zu vereiteln.

Freilich bleiben solche Deutungen spekulativ. Zum einen ist fraglich, ob die CSU-Wähler im Interesse der CDU/CSUFraktion im Bundestag tatsächlich davon absehen, der CSU in Bayern ihre Stimme zu geben. Zum anderen gilt bei der Diskussion um strategische Stimmabgaben $\mathrm{zu}$ bedenken, dass in der Regel die Steuerbarkeit der gewünschten Auswir- 
kung ungewiss bleibt. Im Fall des der Entscheidung des $B$ VerfG zu Grunde liegenden Sachverhalts war es erst die Nachwahl im Wahlkreis Dresden I, die negative Stimmgewichte kalkulierbar machte. ${ }^{24}$

Das fiktive Szenario illustriert jedenfalls, dass auch auf der Ebene der Oberzuteilung Fälle auftreten können, die dem negativen Stimmgewicht ähneln. Dies wird deshalb im Folgenden in die Lösungsoptionen mit einbezogen. Die Unterschiedszahl zwischen Direktmandaten und Verhältnissitzen gibt den Optionen Struktur. Man kann (mit $\$ 6$ Abs. 5 Satz 1 BWahlG) die Zahl der Direktmandate als fest ansehen und verhindern, dass die Zahl der Verhältnissitze darunter fällt (III.). Oder man hält an den Verhältnissitzen fest und deckelt damit die Zahl der Direktmandate (IV.). Schließlich kann man auch separate Berechnungen pro Bundesland durchführen, um so landeslistenübergreifende Sitztransfers zu unterbinden (V.).

\section{Personenwahl vor Verhältniswahl: Parlamentsvergrößerung und Augsburger Zuteilungsverfahren (Option P)}

Das direktmandatsbedingte Divisorverfahren mit Standardrundung (Augsburger Zuteilungsverfahren) bietet eine erste Möglichkeit, um negative Stimmgewichte bei den Unterzuteilungen an die Landeslisten der Parteien auszuschließen. Von dem Sitzkontingent, das die Parteien auf Bundesebene bekommen, erhalten ihre Landeslisten mindestens so viele Sitze, wie sie Wahlkreissieger vorweisen. Die Bestimmung des Wahlschlüssels ist an diese Zusatzbedingung anzupassen, was rechnerisch kein Problem darstellt. Die Komponenten von Personenwahl und Verhältniswahl werden dadurch so verbunden, dass das bessere Ergebnis - die Direktmandate oder die angepassten Verhältnissitze - zum Tragen kommt. ${ }^{25}$

Bei der Wahl 2009 wären über die 173 CDU-Direktmandate hinaus der CDU keine weiteren Sitze zugefallen. Die bundesweite Verhältnisrechnung gibt der CDU 173 Verhältnissitze (siehe Schaubild 1), was exakt gleich der Zahl ihrer Wahlkreissiege ist. Die 21 CDU-Mandatsträger, die jetzt über Landeslisten in den Bundestag eingezogen sind, gäbe es erst gar nicht. Bundestagspräsident Lammert wäre ohne Mandat geblieben wie auch Ministerin von der Leyen, um die Konsequenzen einmal zu personifizieren. ${ }^{26}$

Die direktmandatsbedingte Variante stellt allerdings nur eine Minimalreparatur dar, die das Auftreten negativer Stimmgewichte - wie vom BVerfG verlangt - bei den Unterzuteilungen heilt. Vor der Wahl 2009 waren die bundesweiten Oberzuteilungen immer frei von der diskutierten Problematik und die Minimallösung wäre vertretbar gewesen, der Bundestag konnte sich aber nicht dazu durchringen. Nach der Wahl 2009 stellt sich die Lage anders dar. Die erstmalig angefallenen Überhangmandate der CSU und die

24 Das $B$ VerfG hebt diese Besonderheit selbst hervor; $B \operatorname{VerfGE} 121,266$ (299); sehr deutlich Bebnke KritV 1/2010, 1 (18).

25 Pukelsheim DVB1. 2008, 889 (894). Ein ergebnisgleicher Rechenweg wurde schon 1996 in einem Gesetzentwurf der Fraktion Bündnis 90/Die Grünen aufgezeigt; siehe BT-Drs. 13/5575 vom 24. 9. 1996. Schon seit 1998 wird die direktmandatsbedingte Variante für die Wahl zum Schottischen Parlament praktiziert, allerdings in Verbindung mit dem Divisorverfahren mit Abrundung (D’Hondt/Hagenbach-Bischoff) an Stelle des hier relevanten Divisorverfahrens mit Standardrundung; siehe www.opsi.gov.uk/acts/ acts1998/ukpga_19980046_en_2.

26 Das wäre wahrscheinlich eher nicht eingetreten, da bei Option P die Prominenz wohl darauf bestanden hätte, in einem sicheren Wahlkreis zu kandidieren. auf Bundesebene in greifbare Nähe gerückten Überhangmandate der CDU rufen nach einem ergänzenden Eingriff in die Oberzuteilung.

Eine systemnahe Korrekturmöglichkeit bestünde darin, die Bundestagsgröße über die Anfangssitzzahl 598 hinaus heraufzusetzen, bis alle Überhangmandate abgebaut sind. ${ }^{27}$ Bei der vergangenen Wahl 2009 wäre der Überhang an Sitzen in der Oberzuteilung ab einer Bundestagsgröße von 641 Sitzen beseitigt. Bei dieser Größe entfielen auf die CDU 186 Mandate (Divisor 63 500). Dementsprechend verblieben der CDU über die 173 Direktmandate hinaus 13 Sitze, die aus Landeslisten zu besetzen wären. Bundestagspräsident und Ministerin wären gerettet.

Die zweigleisige Lösung, Probleme in der Oberzuteilung durch eine Vergrößerung des Bundestags zu umgehen und Probleme in den Unterzuteilungen durch die direktmandatsbedingte Variante des Divisorverfahrens mit Standardrundung zu beseitigen, sei als Option P bezeichnet, da die Personenwahlkomponente dominiert. Zwei Einwände erscheinen bedenkenswert.

Erstens ließe sich einwenden, dass ein Bundestag mit 641 Sitzen zu groß sei. Aber um am Ende mit weniger Sitzen heraus zu kommen, müsste man nur am Anfang die Ausgangszahl von 598 Sitzen (und 299 Wahlkreisen) herabsetzen. Unterstellt man ein gleichbleibendes Wahlverhalten, wären in der Wahl 2009 bei anfänglichen 550 Sitzen (und 275 Wahlkreisen) am Schluss um die 600 Sitze zusammen gekommen, bei anfänglichen 500 Sitzen (und 250 Wahlkreisen) nur etwa 550.

Der erste Verfahrensteil der Option P, den Bundestag gegebenenfalls zu vergrößern, vermeidet Überhangmandate nur bei der Oberzuteilung, nicht aber bei den Unterzuteilungen. Selbst bei einer Ausgangszahl von 641 Sitzen, bei der in der Oberzuteilung alle Direktmandate von der Verhältnisrechnung getragen werden, kommt es nach geltender Gesetzeslage bei der Unterzuteilung von dann 186 Sitzen an die CDU-Landeslisten noch zu insgesamt 16 Überhangmandaten. Der Bundestag würde also $657(=641+16)$ Mitglieder umfassen. Nach derzeitiger Regelung würden zum Beispiel der nordrhein-westfälischen CDU-Landesliste 49 der 186 Sitze zugeteilt, unberührt von den anderswo ins Leben gerufenen Überhangmandaten.

An dieser Stelle käme ein zweiter Einwand ins Spiel. Die direktmandatsbedingte Variante, der zweite Verfahrensteil von Option P, kennt keine Überhangmandate. Von den 186 CDU-Verhältnissitzen werden 173 für die Wahlkreissieger reserviert. Nachfolgend kann nur über 13 Sitze verfügt werden. Die nordrhein-westfälischen CDU-Stimmen bekommen nunmehr 41 Sitze zugeteilt. Dies macht acht Sitze weniger als nach geltendem Gesetz. Zur Ausstattung der vorherigen 16 Überhangmandate werden acht $(=49-41)$ allein von der nordrhein-westfälischen CDU-Landesliste geschultert. Die dortigen CDU-Wähler könnten sich im Vergleich etwa zu den baden-württembergischen CDU-Wählern benachteiligt fühlen.

Hier prallen zwei konkurrierende Gleichheitsansprüche aufeinander. Einerseits steht parteiübergreifend der Gleichheitsanspruch des Wahlvolks im gesamten Wahlgebiet im Raum. Hauptziel ist, dass alle Parteien in einem möglichst den Stimmenzahlen angenäherten Verhältnis im Bundestag vertreten sind. ${ }^{28}$ Die Erfolgswertgleichheit bei der (Ober-) Zuteilung der Sitze an die Parteien wird durch das beschriebene Verfahren gesichert. Dies wiegt umso schwerer, als der

27 Pukelsheim/Maier ZParl 2008, 312 (315).

$28 B \operatorname{VerfGE~121,266(296).}$ 
Bundestag das Repräsentativorgan auf Bundesebene ist und föderale Aspekte eine nachgeordnete Rolle spielen.

Als weiteres Ziel können im Wahlsystem auch Belange des föderalen Proporzes berücksichtigt werden. ${ }^{29}$ Jede Parteianhängerschaft unter sich (im obigen Beispiel die CDUWähler) sollte über die diversen Landeslisten hinweg einen gleichen Erfolgswert für ihre Stimmen erzielen. Die Erfolgswertgleichheit bei den (Unter-)Zuteilungen an die Parteilandeslisten erscheint als eine Differenzierung, die dem Hauptziel nachgeordnet ist.

Die direktmandatsbedingte Variante beschneidet die Erfolgswertgleichheit in den Unterzuteilungen, um die Verhältnissitze an die mit den Erststimmen gewonnenen Direktmandate anzupassen und um negative Stimmgewichte zu vermeiden. Dies ist eine Möglichkeit, die vom Bundeswahlgesetz gewollte Verbindung von Personenwahl und Verhältniswahl herzustellen.

\section{Verhältniswahl vor Personenwahl: Bayerisches Vorbild 1954 (Option V)}

Als Option V sei die Möglichkeit diskutiert, die Verhältnissitze einer Landesliste als Obergrenze festzulegen, wie viele Sitze für die Wahlkreissieger zur Verfügung stehen. ${ }^{30}$ Reichen die Verhältnissitze nicht aus, kommen die Wahlkreissieger mit den geringsten Erststimmenzahlen nicht mehr zum Zuge. Diese Regelung praktizierte Bayern für die Landtagswahl 1954. ${ }^{31}$ Der Bayerische VerfGH beurteilte sie als verfassungsgemäß. ${ }^{32}$ Logik und Argumentation des Urteils bleiben auch im Licht der heutigen Rechtsprechung aktuell.

Die Regelung, bei unzureichender Zahl von Verhältnissitzen in einem Bundesland den schwächsten Wahlkreissiegern keinen Sitz zuzuteilen, lässt sich gleichermaßen auf Ober- und Unterzuteilungen anwenden. Im Endergebnis wird die Ausgangszahl von 598 Gesamtsitzen makellos eingehalten. Wenn die Zweitstimmenzahlen unverhältnismäßig hinter den Erststimmenzahlen zurück bleiben, schlagen die Konsequenzen auf die Personenwahlkomponente durch. ${ }^{33}$ Dies ist eine zweite Möglichkeit, die vom Gesetz gewollte Verbindung zwischen Personenwahl und Verhältniswahl herzustellen.

Option V geht mit dem geringsten rechnerischen Eingriff einher. Die Verhältnisrechnung läuft ab wie bisher, ohne dass Zusatzbedingungen erforderlich sind. Die Oberzuteilung wie auch alle Unterzuteilungen gehorchen dem Prinzip der Erfolgswertgleichheit der Zweitstimmen. An die Verhältnissitze, die sich so ergeben, wird das Personenwahlergebnis angepasst. Negative Stimmgewichte sind passé. Der politische Eingriff der Option ist dagegen immens, wird doch der Grundsatz aufgegeben, dass jeder Wahlkreis stets durch einen Abgeordneten im Bundestag vertreten ist. Option V kann so gedeutet werden, dass sie sich am Verursacherprinzip orientiert. Personen- und Verhältniswahl werden in denjenigen Bundesländern miteinander verbunden, wo sie auseinander zu laufen drohen. Anstatt in Baden-Württemberg zehn Überhangmandate entstehen zu lassen, die aus Nord-

$29 B \operatorname{VerfG} E$ 121, 266 (302).

30 Der Vorschlag wurde schon 1902 von Geyerhabn (Fn. 19) gemacht. \5 BWahlG bedarf dann eines entsprechenden Vorbehalts.

31 Unterpaul, Die Grundsätze des Landeswahlrechts nach der Bayerischen Verfassung im Lichte der Entwicklung von 1946 bis 1989, 1992, S. $80-83,176-179$.

32 BayVerfGH 7 (1954), 99 (104f.).

33 Bei der Wahl 2009 entfielen auf die 24 Wahlkreissieger, die in Überhangländern die schwächsten Erststimmenerfolge vorweisen, im Durchschnitt grob ein Drittel der Erststimmen (38 Prozent).
rhein-Westfalen und anderswo abgezogen werden, findet die Reparatur im Ländle selbst statt. Zudem stärkt Option V die selbstregulierenden Kräfte des Systems. Zukünftig wäre es im Interesse nicht nur der Wähler, sondern auch der Kandidaten und Parteien, möglichst gleichgroße Wahlkreise einzurichten. Zu kleine Wahlkreise bergen die Gefahr in sich, dass die Wahlkreissieger wegen zu geringer Erststimmenerfolge nicht mehr zum Zuge kommen. ${ }^{34}$

\section{Erhalt der Überhangmandate: Föderales Eigenständigkeitsmodell (Option F)}

Eine Vermeidung negativer Stimmgewichte ist auch ohne Abschaffung von Überhangmandaten möglich. Denkbar ist eine sechzehnfache, separat für jedes Bundesland vorgenommene Auswertung der Wahlergebnisse. Bei separaten Sitzzuteilungen pro Bundesland können Überhangmandate in einem Land nicht auf ein anderes ausstrahlen. Negative Stimmgewichte können nicht mehr auftreten.

Bei dieser Option muss allerdings klar sein, wie viele der Sitze auf jedes Bundesland entfallen. Die Sitzkontingente könnten in der Mitte einer Legislaturperiode von der Wahlkreiskommission zusammen mit der Wahlkreiseinteilung im Verhältnis der Bevölkerungszahlen bestimmt werden.

Problematisch erscheint der Widerspruch, mit dem dieses aufgebrochene Wahlsystem dem unitarischen Charakter des Bundestags entgegen steht, ${ }^{35}$ er wiegt jedenfalls schwerer, als es nach dem geltenden Wahlsystem durch die Berücksichtigung föderaler Aspekte der Fall ist. Zudem drohen zwei Komplikationen, die im Grunde Option F als eine vertretbare Alternative verwerfen. ${ }^{36}$ Erstens kann ein Verhältniswahlsystem der Erfolgswertgleichheit der Wählerstimmen nur nahe kommen, wenn es genügend viele Sitze gibt, um Verhältnismäßigkeit zu ermöglichen. Aus umfangreichen Untersuchungen ergibt sich als Faustregel, dass mindestens doppelt so viele Sitze verfügbar sein müssen, wie es Parteien im Parlament gibt. ${ }^{37}$ Bei fünf Parteiblöcken im Bundestag wäre das Saarland mit acht Sitzen ein kleiner Problemfall, Bremen mit fünf ein großer. ${ }^{38}$ Kleinen Wahldistrikten kann durch die Schaffung von Wahlkreisverbänden entgegen gewirkt werden. Etwa könnten das Saarland und Rheinland-Pfalz einen Verband bilden ${ }^{39}$ und Bremen mit Niedersachsen einen anderen. Option F, die die föderale Struktur hofieren soll, würde stattdessen die Kleinstaaterei an den Pranger stellen.

Zweitens zwingt die Option das Wahlvolk dazu, sich ein strategisches Kalkül der Stimmenabgabe anzueignen. Zweitstimmen für eine überhängende Landesliste sind verloren.

\footnotetext{
34 Bei der Wahlkreiseinteilung wäre eine Herabsetzung der Abweichungsschwellen von 15 und 25 Prozent in $\$ 3$ Abs. 3 Satz 3 BWahlG auf 10 und 15 Prozent nach den Empfehlungen der OSZE sinnvoll; vgl. Wahl zum Deutschen Bundestag, 27. September 2009, Bericht der OSZE/ ODIHR-Wahlbewertungskommission, Warschau, 14. Dezember 2009, Abschnitt V.B; www.osce.org/documents/odibr-el/2009/12/42097_de.pdf.

35 H. Meyer DVBl. 2009, 137 (140).

36 Das BVerfG bewertet das föderale Anliegen der Ausgestaltung des Wahlsystems zwar als verfassungslegitimes, aber nicht als sehr starkes Ziel; vgl. $B$ VerfGE 121, 266 (303 ff.)

37 Pukelsheim/Maier/Leutgäb NWVBl. 2009, 85 (88).

38 Das Problem zu kleiner Wahldistrikte, mit dem sich die schweizerischen Kantone regelmäßig konfrontiert sehen, kann durch das doppeltproportionale Divisorverfahren mit Standardrundung gelöst werden (Zürich, Schaffhausen, Aargau); siehe Pukelsheim/Schubmacher AJP/PJA 2004, 505 (512). Was gut zur schweizerischen Tradition passt, ist allerdings nicht automatisch auch attraktiv aus deutscher Sicht. Zudem wäre die doppeltproportionale Variante zu modifizieren, um die mit den Erststimmen gekürten Wahlkreissieger unterzubringen.
}

39 So Isensee DVBl 2010, 269 (275). 
Der Stimmzettelaufdruck, dass die Zweitstimme die maßgebende Stimme für die Verteilung der Sitze insgesamt auf die einzelnen Parteien sei, wird unhaltbar. Bei der Wahl 2009 hätte die CDU in Baden-Württemberg ihre (Direkt-) Mandate auch ohne jede Zweitstimme erlangt, ebenso die CSU in Bayern. Insgesamt wäre knapp ein Fünftel der zuteilungsberechtigten Zweitstimmen wirkungslos verpufft. ${ }^{40}$ Option $\mathrm{F}$ wäre insofern eine Einladung, die zwei Stimmen stärker strategisch einzusetzen als bislang, ${ }^{41}$ was rechtlich nach überwiegendem Verständnis der Wahlfreiheit durchaus zulässig wäre. Wollte man einem massiven strategischen Stimmensplitting entgegenwirken, wäre der Übergang zu einem Einstimmensystem zu erwägen.

\section{Mehrheitswahl und Mythologie}

Die Bewertung jeglicher Handlungsoptionen hängt wesentlich von der Ausformung bzw. Deutung des gesetzlichen Ziels ab, das Wahlsystem nach den Grundsätzen einer mit der Personenwahl verbundenen Verhältniswahl zu gestalten. Auch das BVerfG hat über die Jahrzehnte zur Konfusion beigetragen. Dies reicht von Formulierungen, es handle sich um eine Mehrheitswahl, „die ... der proportionalen Sitzverteilung vorgeschaltet ist“,42 bis zu einem „Verhältnisausgleich, der hinter eine Mehrheitswahl im Wahlkreis gesetzt wird“.43 Andere Entscheidungen lassen Sensibilität für die Systemgestaltung erkennen, wenn es dort heißt, „dass bei der Auswahl der Kandidaten Elemente der Mehrheitswahl vorgeschaltet sind“. ${ }^{44}$

Die tradierte Verkündigung, dass die Personenwahlkomponente einer reinen Mehrheitswahl gleich komme, wird von Lenskit5 äußerst gehaltvoll entmythologisiert. Bei einer Mehrheitswahl gewinnt nur einer, nämlich wer die meisten Stimmen bekommt. ${ }^{46}$ Beim bundesdeutschen Wahlsystem gewinnen aber vielfach auch zweite, dritte oder gar weitere Wahlkreisbewerber. Sie alle ziehen in den Bundestag ein, einer als Wahlkreiskönig und die anderen über Landeslisten. Eine Personenwahl, die mit der Verhältniswahl verbunden ist, ist solange keine reine Mehrheitswahl, wie sich Kandidaten sowohl um Wahlkreis- als auch um Listenmandate bewerben. Sie scheidet nicht die Mehrheit von der Minderheit. Nur ein Drittel der Wahlkreise (97) im amtierenden Bundestag sind allein durch ihren Wahlkreissieger vertreten, 117 durch zwei Abgeordnete, 64 durch drei, 19 durch vier und die Wahlkreise 252 und 279 (Augsburg-Stadt und Pforzheim) sogar durch alle fünf. ${ }^{47}$ Der Personenwahlkomponente kommt insofern wohl eher eine psychologische Funktion zu. Sie macht deutlich, dass viele Wähler durch wenige Mandatsträger repräsentiert werden. Diese menschliche Dimension strahlt auch auf die Zweitstimme aus und diese Ausstrahlung ist nicht gering zu schätzen. Die Rolle der Personen würde in einem reinen Verhältniswahlsystem mit starren Listen weit

\footnotetext{
40 In Zahlen: 7642 109/40764288 = 19 Prozent.

41 Eindringlich Behnke KritV 1/2010, 1 (25).

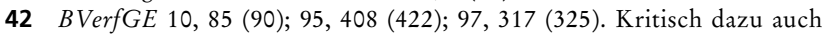
Noblen ZParl 2009, 179 (186).

$43 B \operatorname{VerfGE} 6,84$ (90).

44 BVerfGE 31, 127 (129); 66, 291 (304).

45 Lenski AöR 134 (2009), $473 \mathrm{ff}$.

46 Allgemein zum Mehrheitsprinzip jüngst Krüper www.zjs-online.com/ dat/artikel/2009_5_223.pdf.

47 Hinzu kommen 13 Listenbewerber, die in keinem Wahlkreis kandidiert haben; Der Bundeswahlleiter (Hrsg.), Wahl zum 17. Deutschen Bundestag am 27. September 2009, Heft 3, Endgültige Ergebnisse nach Wahlkreisen, 2009, S. 206-239. Für die 16. Legislaturperiode siehe Lenski AöR 134 (2009), 473 (594).
}

weniger betont. Dass darüber hinaus Parteien hinzutreten, um beim Massengeschäft demokratischer Volkswahlen als vermittelnde Institutionen mitzuwirken, steht diesem Verständnis nicht entgegen.

Wer das Heil der Demokratien im Mehrheitswahlrecht erblickt, wird als Reparaturoption das "Grabensystem“ favorisieren. Beim Grabensystem wird eine Hälfte des Bundestags durch reine Mehrheitswahl bestimmt, die andere durch reine Verhältniswahl. $48 \mathrm{Da}$ dann der Verhältnisrechnung nur halb so viele Sitze wie derzeit zur Verfügung stehen, halbiert das Grabensystem die Repräsentation der Wählerschaft kleinerer Parteien, die keine Wahlkreissieger küren können. Verfassungsrechtlich bedeutsamer dürfte sein, dass mit Blick auf die Wahlgrundsätze gleichgroße Wahlkreise eingerichtet und mehrheitswahltaugliche Nachrückerregelungen geschaffen werden müssen, weder das eine noch das andere ist derzeit gegeben. Zudem geht jede Mehrheitswahl unweigerlich einher mit Wahlabsprachen, die 1918 als Grund für ihre Abschaffung galten: „Unleugbare Vorteile [eines Verhältniswahlsystems] sind auch der Wegfall jeder Nötigung zu unnatürlichen Wahlbündnissen“.49

\section{Schlussfolgerungen}

Im Ergebnis stellen sich die föderale Option $\mathrm{F}$ wie auch das Grabensystem als zu problembeladen dar, um als empfehlenswerte Handlungsalternativen fortzubestehen. Die Optionen $\mathrm{P}$ und $\mathrm{V}$ erscheinen dagegen als gangbar. Beide schaffen eine wirksame Verbindung zwischen der Personenwahl und der Verhältniswahl, wie das Bundeswahlgesetz es will.

Option P steht dem derzeitigen Wahlsystem näher. Die nach alter Art bei den Unterzuteilungen ins Leben gerufenen Überhangmandate würden von den überhangfreien Landeslisten derselben Partei alimentiert. Allerdings bestünde für die Parteipolitiker des Landes, deren Liste überhängt, kaum Veranlassung, daran etwas zu ändern. Wähler, die durch Stimmensplitting bewusst auf Überhangmandate hinarbeiten, könnten ihr Verhalten in dem Bewusstsein beibehalten, nicht nur die Repräsentation der Partei ihrer Wahl, sondern auch die ihres Bundeslandes zu verstärken.

Option V bietet das größere Potential, auch langfristig Bestand zu haben. Sie beinhaltet für die Parteipolitiker eines Landes Anreize zu selbstkorrigierenden Aktionen, da die Anpassungseffekte im betroffenen Land selbst spürbar würden. ${ }^{50}$ Natürlich ist es für die Wähler enttäuschend, dass beide Stimmen ohne Wirkung bleiben können. Die 24 schwächsten Wahlkreissieger, die bei der Wahl 2009 in Überhangländern zusammen kamen, wurden von 1141487 Erststimmen getragen, die bei Option $\mathrm{V}$ unwirksam blieben. Dem steht allerdings mit 2506862 mehr als das Doppelte an Zweitstimmen zur Seite, die - obwohl maßgeblich - mangels Zuteilungsberechtigung ebenfalls keine Wirksamkeit entfalten. Dies sollte man als zwei Seiten derselben Medaille werten. Eine mit der Personenwahl verbundene Verhältniswahl bleibt eine hybride Konstruktion, die nicht frei von Nachteilen ist, deren Vorteile aber bei weitem überwiegen.

Die Verbindung zwischen den Systemkomponenten Personenwahl und Verhältniswahl, die in den Optionen P und V

48 Unter der Bezeichnung gemischtes Wahlsystem schon bei Erdmannsdörffer, Ein neues Wahlgesetz - Vorschläge zur Schaffung eines „gemischten Wahlsystems“, 1919, S. 36. Kritisch Mabrenholz, in: Festschrift für Winfried Hassemer, 2010, S. 111 (118).

49 RT-Drs. 13/1288, II. Session 1914/18 (11).

50 Pukelsheim KritV 2000, 76 (97). 
hergestellt wird, befreit auch das Nachrücken aus der Landesliste vom Odium eines systemfremden Bruchs. Eine Unterschiedszahl, die ausdrückt, dass in einem Land einer Partei mehr Direktmandate zufallen als Verhältnissitze, kann bei keiner der beiden Optionen zu Stande kommen. Das Nachrücker-Urteil des $B \operatorname{VerfG}$ wird obsolet. ${ }^{51}$ Zudem könnten Nachwahlen wie 2005 die im Wahlkreis Dresden I durch eine Nachrückerregelung aus der Liste umgangen werden.

Bei beiden Optionen $\mathrm{P}$ und $\mathrm{V}$ wird darüber hinaus das Konstrukt des doppelten Stimmenerfolgs hinfällig, das im derzeitigen System ein zwielichtiges Dasein führt. Manche Stimmen werden wegen eines drohenden Doppelerfolgs kassiert, andere nicht. ${ }^{52}$ Die verbundene Auswertung der zwei Stimmen bei den Optionen P und V bietet allen Wählern die Chance, mit zwei Stimmen zum Endergebnis beizutragen. Es gäbe keinen Anlass mehr, den freien Gebrauch der beiden Stimmen im Gesetz zu beschneiden.

Wahlgesetze sind Entscheidungen des Parlaments in eigener Sache. ${ }^{53}$ Es droht stets die Gefahr, dass sie von der Mehrheit der Abgeordneten zur Sicherung ihrer Mehrheit genutzt werden. Das $B \operatorname{VerfG}$ hat mit Weitblick die Frist für die Än-

$\overline{51 B \operatorname{Verf} G E}$ 99, 1.

52 Pukelsheim DVB1. 2008, 889 (893).

53 v. Arnim JZ 2009, $813 \mathrm{ff}$. derung des Wahlgesetzes in die Mitte der (regulären) Amtsperiode gelegt, weil belastbare Prognosen für die Wahlen zum 18. Bundestag dann noch nicht vorliegen können. Unabhängig davon ist die Ausgestaltung des Wahlsystems eine Grundsatzentscheidung von materiellem Gewicht, die einer breiten politischen Diskussion nicht nur im Parlament und in den politischen Parteien, sondern auch in der Gesellschaft bedarf. Dass diese Diskussion bislang nicht geführt wurde, jedenfalls nicht hinreichend, ist ein Versäumnis, das ein recht schlechtes Bild vom demokratischen Gemeinwesen in Deutschland gibt. Dem Gesetzgeber verbleibt nun nur noch weniger als ein Jahr, um eine Wahlrechtsänderung auf den Weg zu bringen, die dauerhaft dem wichtigsten Akt der Legitimationsvermittlung Gestalt gibt. Dabei sollten sich alle Akteure bis hin zum gegebenenfalls überprüfenden $B V \operatorname{erf} G$ darüber im Klaren sein, dass die drei unterschiedlichen verfassungsrechtlichen Eckpunkte, an denen sich das Wahlrecht ausrichten muss, nicht alle im gleichem Maße verwirklicht werden können - sie geben nur die Form des Dreiecks vor, nicht aber dessen inhaltliche Ausgestaltung. Die von der Verfassung bewusst dem Gesetzgeber überlassene Entscheidung, wie das Wahlrecht im Einzelnen ausgestaltet ist, impliziert insoweit auch, dass die verfassungsrechtliche und gerichtliche Kontrolle auf die Grundsätze des Wahlsystems beschränkt bleibt.

Professor Dr. Axel Metzger, LL.M. (Harvard), Hannover*

\section{Perspektiven des internationalen Urheberrechts - Zwischen Territorialität und Ubiquität}

\begin{abstract}
Das internationale Urheberrecht sieht sich gegenwärtig erheblichem Veränderungsdruck ausgesetzt. Auf der einen Seite stehen die Medienindustrien, welche ihre bisherigen Geschäftsmodelle nur mit Schwierigkeiten an die Gewohnheiten der Nutzer im Internet anpassen können und nach einer Verstärkung des Rechtsschutzes rufen. Auf der anderen Seite fordert eine Koalition aus Nutzern und Internetdiensten die Beibehaltung des weitgehend ungehinderten Zugangs zu Inhalten und Technologien. Der folgende Beitrag beleuchtet vor diesem Hintergrund die aktuellen Entwicklungen im Recht der Staatsverträge und im Urheberkollisionsrecht.
\end{abstract}

\section{Aufstieg und Fall der Konventionen - Von RBÜ zu ACTA}

\section{Ausgangslage im 19. Jahrhundert}

Die aktuellen Entwicklungen im internationalen Urheberrecht lassen sich nur vor dem Hintergrund der Rechtsentwicklung seit dem Abschluss der ersten Staatsverträge begreifen. Als Ausgangspunkt bietet sich das Jahr 1886 an, am Vorabend der Unterzeichnung der ersten und bis heute

\footnotetext{
* Der Autor ist Inhaber des Lehrstuhls für Zivilrecht, Geistiges Eigentum, Informationstechnologierecht und Internationales Privatrecht an der Juristischen Fakultät der Leibniz Universität Hannover, Institut für Rechtsinformatik. Der Beitrag ist die Schriftfassung seiner Antrittsvorlesung vom 16.3.2010, ergänzt um einzelne Nachweise.
}

wichtigsten internationalen Konvention im Urheberrecht, der Berner Übereinkunft. Die Behandlung von Urheberrechtsstreitigkeiten mit Auslandsbezug war im ausgehenden 19. Jahrhundert von zwei Prinzipien geprägt, der Territorialität des Urheberrechts und der exklusiven Schutzgewährung für die eigenen Staatsangehörigen.

Trotz der Abschaffung der vom Landesherren erteilten Privilegien für Verleger und Autoren ${ }^{1}$ und der allgemeinen gesetzlichen Anerkennung des Schutzes aller Werke der Literatur, Wissenschaft, Musik und Kunst behielt der größere Teil der führenden Kultur- und Wirtschaftsnationen das Prinzip bei, wonach jeder Staat nur Urheberrechte für sein eigenes Territorium anerkennt. Handlungen im Ausland konnten demnach nicht zur Verletzung inländischer Rechte führen, es sei denn, die Gesetze definierten auch bestimmte extraterritoriale Handlungen als Verletzungen des inländischen Rechts. ${ }^{2}$ Dieser Grundsatz lässt sich für Deutschland, ${ }^{3}$

\footnotetext{
1 Siehe hierzu Gieseke, Die geschichtliche Entwicklung des Urheberrechts, 1957, S. 23 ff.; Schack, Urheber- und Urhebervertragsrecht, 5. Aufl. 2010, S. 53 ff.; Vogel, in: Schricker, Urheberrecht, 3. Aufl. 2006, Einleitung Rn. $52 \mathrm{ff}$.

2 Vgl. etwa $\$ \int 18,22$ Gesetz (Norddeutscher Bund) betreffend das Urheberrecht an Schriftwerken, Abbildungen, musikalischen Kompositionen und dramatischen Werken vom 11.6.1870 und hierzu die Kommentare von Endemann, 1871, und Dambach, 1871. Zum Ausnahmecharakter dieser Regelungen siehe Beckstein, Einschränkungen des Schutzlandprinzips, 2010, S. $39 \mathrm{f}$

3 Siehe für Deutschland Kobler, Urheberrecht an Schriftwerken und Verlagsrecht, 1907, S. $213 \mathrm{ff}$.
} 
